$\mathrm{OH}_{3}+$ ion. The sharpening of bands at low temperatures and other features may find application in certain analytical work; but this may involve some experimental problems, and the formation on solidification of an amorphous glass rather than a crystalline mass may be vital.

Attempts to study reaction intermediates and radicals through their spectra have not, so far, been entirely successful. D. F. Hornig (Brown University) described a new approach in which shock waves have been used to excite molecular species to a 'temperature' equivalent to that of reactions or combustion processes. The time required to induce this excitation--some microseconds-may be much less than the reaction times, and it is hoped, therefore, from spectral emission or absorption to study the subsequent kinetic behaviour of the species. Very fast photographic and photoelectric methods have been used to record spectra of certain radicals produced by passing shock waves into substances such as cyanogen halides or methyl halides, and the progress of such work should be interesting. The identification of radicals in flames by resolution of their vibrationalrotational emission bands was achieved recently by Plyler, Benedict and Silverman. G. R. Wilkinson, M. A. Ford and W. C. Price (King's College, London) have now made similar measurements on emission bands in the region 1-5.5 $\mu$ of simple molecules and radicals excited by a radio-frequency discharge in hydrogen halides, methane, water vapour and other gases. This method has definite advantages over absorption measurements in that band sequences are more fully developed, and both the rotational and vibrational constants can be measured conveniently. The results of recent absorption studies on a number of hydrocarbons using very high resolving-power were also outlined by $\mathbf{H}$. W. Thompson. From all these data, more precise values are being obtained, not only for the main molecular rotational constants but also for the centrifugal stretching-coefficients and anharmonic vibration factors.

Among all the above fairly conventional themes, a new note was struck at the meeting - the study of nuclear magnetic resonance and R. E. Richards (Oxford) illustrated its value by many examples of practical importance. The spin of a charged nucleus gives rise to a magnetic effect, and the associated magnetic vector can be oriented in different ways in an applied magnetic field. These orientations correspond to different quantized energy-levels, between which transitions can take place. For fields of 10 kilogauss the absorption frequency lies in the radio region, 'resonance' occurring between the two levels and different nuclei absorbing at characteristically different frequencies. - When interaction occurs between the nuclei and their surroundingsto be exact between their respective magnetic effects -the absorption lines broaden in ways which depend upon the internuclear distances and other structural features. Thus groups such as $\mathrm{CH}_{3}, \mathrm{CH}_{2}$ or $\mathrm{OH}$ can be characterized. Information of the most varied kind, both qualitative and quantitative, can now bo obtained, and it is certain that much will result from this new highly sensitive method.

It is clear that there is intense activity in this field. The difficult task of setting up a punched-card system for reference spectra was also discussed, and it is hoped that the committee set up several years ago in Britain to consider this matter may shortly be able to institute a scheme.

H. W. Thompson

\section{FULMER RESEARCH INSTITUTE, STOKE POGES OPEN DAY}

$\mathrm{A}$ $\mathrm{N}$ informal visit was made to the Fulmer Research Institute on November 2 by H.R.H. the Duke of Edinburgh on the occasion of an open day held to mark the opening of the new Mechanical Testing Laboratory of the Institute. After a tour of the laboratories lasting for an hour and a half, during which he pulled the first test-piece in the new 50-ton tensile-testing machine of the Laboratory, the Duke had luncheon at the Institute together with some three hundred guests representing industry and research. In his speech he said that he had always been convinced that the research business was a good investment and that he was interested to have practical proof in an establishment which, without outside support, does indeed make a profit entirely on research. After luncheon the laboratories were open to the other guests, and the scientific exhibits showed in detail some of the work of the Institute during its first seven years of existence.

Following the invention of the catalytic distillation process for refining of aluminium, Dr. P. Gross and his colleagues demonstrated similar studies applied to the extraction of titanium from impure titaniumbearing materials. Laboratory exhibits showed that titanium can be removed from an impure titaniumbearing alloy by reaction with a high-valency halide to form a halide of lower valency in the vapour phase. Disproportionation of the lower-valency halide then gives a deposit of pure metallic titanium with reformation of the higher-valency halide. In connexion with the work on the purification of both aluminium and titanium, an intensive study is in progress of the reactions which may be involved in these processes, and an important feature of the work is the determination of the heats of formation of metallic halides. The apparatus, which includes dry boxes for handling deliquescent halides and a calorimeter made in the laboratory workshops, was on view. The heat of formation of aluminium fluoride has recently been re-determined by Dr. Gross, and a new value, differing appreciably from that previously accepted, has been established and the results published. The heat of formation of titanium tetrachloride has also recently been determined, but the results obtained are at present confidential to the sponsor. Further work is in hand on the heats of formation of other halides. A recent step forward in Dr. A. H. Sully's work on chromium and its alloys has been the preparation of a ductile form of chromium. Flakes of electrodeposited chromium which are capable of being bent were on view.

In the Physics Section apparatus was set up for studying by $\mathrm{X}$-ray diffraction the structure of liquid metals. This involved the use of Geiger counters in plotting the variation in intensity of $\mathrm{X}$-ray diffraction from a liquid metal surface. Apparatus for measuring the vapour pressure of reactive metals such as uranium was also in operation. Many novel types of camera and a curved quartz erystal monochromator were included in the apparatus for studying the progress of precipitation hardening and the ensuing structural changes. There was an extensive exhibit of the results of such an investigation on the ageing of aluminium copper alloys.

Dr. H. K. Hardy's studies on the thermodynamics of alloy systems and the mechanism of precipitation 
hardening were also demonstrated, together with the results of recent research on aluminium bronzes and on the aluminium-copper-cadmium alloys. In the Corrosion Laboratory one exhibit indicated the marked influence of directionality of structure on the corrosion and stress-corrosion behaviour of highstrength aluminium alloys. This was correlated with work on the properties of material at the flash line in aluminium alloy forgings where a very high degree of directionality has been observed.

New methods of chemical and spectrographic analysis were demonstrated in the Chemical and Spectrographic Sections. These included a combined chemical and spectrographic method for the estimation of tantalum and niobium in certain soils. Apparatus recently acquired in the experimental foundry includes a vacuum or argon melting furnace in which reactive metals such as uranium and titanium can be arc-melted on to a water-cooled copper hearth. In common with a great deal of other apparatus, this has been constructed in the laboratory workshops. Facilities for metal working included a 60-ton press for powder metallurgical and similar work, a new laboratory rolling mill, a 1-cwt. forging hammer and a small laboratory extrusion press. In. connexion with an investigation on the properties of semi-conductors, a technique for melting and casting rods of pure silicon for subsequent purification by zone melting was demonstrated. An exhibit showed work in progress on the developmont of heat-resisting coatings, and the high-oxidation resistance of steel containing relatively small quantities of silicon and aluminium ( 3 per cent and 1 per cent, respectively) was demonstrated. Apparatus for the study on a pilotplant scale of the process for catalytic distillation of aluminium was on view, together with a wide variety of furnaces for heat treatment, including those with facilities for rapid water quenching from vacuum or special atmospheres.

The new Mechanical Testing Laboratory contains a 50-ton Denison tensile compression machine and a thermostatically controlled creep-testing laboratory with seven Denison tensile creep-testing machines of conventional design and four compression-type creep machines. Machines for testing the creep of strip specimens were also in operation in the creep laboratory. Fatigue tests at both room and elevated. temperature were in progress, and these included reverse bend tests on sheet specimens, rotating cantilever tests and reversed tension (Haigh) fatigue testing. An interesting exhibit in this section dealt with methods of testing and improving the resistance of aluminium and its alloys to fretting and blackening by abrasion. Work in collaboration with the Department of Colloid Chemistry at the University of Cambridge under the direction of Dr. J.H. Schulman has shown that friction between aluminium surfaces can be markedly reduced by the application of substances forming so-called 'penetrated' monomolecular layers.

The activities of the Institute were described in a booklet which shows that, since 1952, thirty-eight papers from the Institute have been published, or are about to be published, by scientific societies. In the library of the Institute it was shown that, while the research reports of the Institute are confidential to sponsors, a large number of reprints of published papers are available, and a particular feature was the large number of translations, which include many scientific papers translated from the original Russian.

\section{STRUCTURE OF VITAMIN $B_{12}$}

\section{A Crystalline Nucleotide-free Degradation Product of Vitamin $\mathbf{B}_{12}$}

IN an earlier paper ${ }^{1}$ we have described a detailed study of the course of hydrolysis of vitamin $B_{12}$ under both acid and alkaline conditions. It was found that under rigorous experimental conditions there were produced 1-amino-2-propanol, ammonia, 5 : 6-dimethylbenziminazole-1- $\alpha$-D -ribofuranoside- 2 '(or 3')-phosphate and a deep red cobalt-containing gum which behaved as a mixture of tetra., pentaand hexa-basic acids in the case of alkaline hydrolysis, or as a mixture of tetra-, penta-, hexa- and hepta-basic acids in the case of acid hydrolysis. The mixture of cobalt-containing acids obtained from the alkaline hydrolysis of the vitamin has now been subjected to further fractionation in order to provide material for chemical degradation and physical examination.

Vitamin $B_{12}$ was hydrolysed with 30 per cent sodium hydroxide at $150^{\circ}$ for $1 \mathrm{hr}$., and the tetra-, penta- and hexa-basic carboxylic acids as well as the accompanying free nucleotide were separated by electrophoresis on Whatman seed-testing paper, ${ }^{1,2}$. Of the three coloured fractions, that corresponding to the hexabasic acid was present in the largest amount. It was converted into its ammonium salt, absorbed on a column of 'Dowex $1 \times 2$ ' resin (2 per cent cross-linking) and eluted with $0.6 \mathrm{M}$ ammonium acetate solution. Several minor fractions were eluted first and were followed by the major band, which was collected separately. It was worked up in the usual way by acidification with hydrochloric acid, extraction into phenol, removal of the inorganic salts from the phenol extract by washing with dilute hydrochloric acid and then water, dilution of the phenol with ether and re-extraction of the red acid into water. After evaporation of the water, the gummy residue was dissolved in aqueous acetone and diluted with ether and kept for several weeks, when a small quantity of red prisms was obtained. This crystalline product was submitted to Dr. D. C. Hodgkin and the elegant crystallographic examination described in the following communication was carried out on one selected crystal. Further dilution of the mother liquors with ether gave fine red needles which were soluble in aqueous acetone but insoluble in either water or acetone alone. Recrystallization was wasteful but could be effected by adding ether cautiously to the aqueous acetone solution, when crystalline material was, obtained from the cloudy solution after removal of some amorphous material precipitated initially. (Found on material dried to constant weight at $54 \% 0.05 \mathrm{~mm}$. : C, $52 \cdot 8$; H, $6 \cdot 3$; $\mathrm{N}, 7 \cdot 85 . \mathrm{C}_{47} \mathrm{H}_{66} \mathrm{O}_{16} \mathrm{~N}_{8} \mathrm{CoCl}$ requires $\mathrm{C}, 53 \cdot 0 ; \mathrm{H}, 6 \cdot 25$; N, 7.9 per cent.) An aqueous solution of the product in dilute nitric acid contained chloride ions.

The ultra-violet absorption spectrum (Figs. 1 and 2) shows a strong resemblance to that of vitamin $\mathrm{B}_{12}$; differences in the $280 \mathrm{~m} \mu$ region are due to the absence of the 5:6-dimethylbenziminazole nucleotide in the hydrolysis product. In the presence of excess cyanide a bathochromic shift of the spectrum is obtained similar to that observed with vitamin $\mathrm{B}_{12}$ and indicates that the chromophore of the hexabasic acid is closely related to that of the parent vitamin. 'The infra-red spectrum, determined on a 'Nujol' mull, showed maxima at $4 \cdot 67,4 \cdot 73$ (weak), 5.77, $5 \cdot 85,6 \cdot 01,6 \cdot 15,6 \cdot 30,6 \cdot 64,7 \cdot 74,8 \cdot 64,8 \cdot 98,9 \cdot 15$, 